\title{
Mobiles Fremdsprachenlernen mit Podcasts
}

\author{
Sylwia Adamczak-Krysztofowicz, Antje Stork und Katarzyna Trojan
}

\begin{abstract}
Zusammenfassung
Mobiles Lernen im Bereich des Fremdsprachenlernens kann in unzähligen Lernsettings und anhand unterschiedlicher Anwendungsszenarien erfolgen. Im Fokus des vorliegenden Beitrags steht das mobile Fremdsprachenlernen mit Podcasts. In dem Beitrag wird zunächst auf theoretische Grundlagen des mobilen Fremdsprachenlernens und den aktuellen Forschungsstand zum Fremdsprachenlernen mit Podcasts eingegangen. Anschliessend wird die Konzeption einer explorativ angelegten Fragebogenstudie erläutert, die am Sprachenzentrum der TU Darmstadt durchgeführt wurde, um zu ermitteln, wie Podcasts von Studenten/-innen zum Fremdsprachenlernen genutzt werden und welche Wünsche es diesbezüglich gibt. Darauf aufbauend werden ausgewählte Befragungsergebnisse in Bezug auf die Nutzung von mobilen Endgeräten und von Podcasts zum Fremdsprachenlernen dargestellt und diskutiert. Zum Schluss werden Konsequenzen für mögliche Anwendungsszenarien innerhalb und ausserhalb des Fremdsprachenunterrichts gezogen.
\end{abstract}

\section{Mobile Foreign Language Learning with Podcasts}

\begin{abstract}
Mobile learning in the field of learning foreign languages may take place in different settings and in various application scenarios. The following article focuses on the mobile learning of foreign languages by means of podcasts. The paper describes firstly the theoretical foundations of mobile foreign language learning and reports the current state of research on using podcasts in learning foreign languages. Subsequently the design of the exploratory questionnaire survey, conducted among students at the Language Centre of TU Darmstadt, will be explained. The survey was carried out in order to find out, how podcasts are used by students to learn foreign languages and what kind of wishes they have in connection with this. Building on this, the selected survey results concerning the use of mobile devices and podcasts to learn foreign languages will be presented and discussed. On this basis, the consequences for possible application scenarios in and outside the foreign language classroom will be drawn here as well.
\end{abstract}


In den Ergebnissen der Trendstudie MMB Learning Delphi erhält mobiles Lernen «mit grossem Abstand die meisten Stimmen», weil mobile Lernapplikationen bei 85\% der befragten Experten «als Umsatzlokomotive des Jahres 2016» (MMB-Institut für Medien- und Kompetenzforschung 2013,2) gelten können. Auch in dem Beitrag von Specht und Ebner $(2011,6)$ ist zu lesen, dass mobiles Lernen «eines der sich derzeit am schnellsten weiterentwickelnden Forschungsgebiete» darstellt. Aus der Perspektive der Fremdsprachendidaktik wurde aber mobiles Lernen bisher wenig erforscht. Derzeit liegen unseres Wissens lediglich einige deutschsprachige Aufsätze (z. B. in dem Basisheft der Zeitschrift Praxis Fremdsprachenunterricht vom Januar 2013) und das Buch von Mitschian (2010) vor, die ausgewählte M-Learning Aktivitäten für den fremdsprachlichen Unterricht darstellen. Empirische Studien im Bereich mobilen Fremdsprachenlernens mit Podcasts stehen auch weiterhin aus. ${ }^{1}$ Im Zentrum unseres Beitrags steht daher die Auswertung unserer explorativ angelegten Fragebogenstudie, die Anfang Februar 2013 am Sprachenzentrum der TU Darmstadt durchgeführt wurde. Anknüpfend an die Frage, wie mobiles Lernen das Fremdsprachenlernen, die Fremdsprachenlerner/innen und die Fremdsprachenlehrer/innen unterstützen kann, wollen wir in unserem Beitrag die mobile Mediennutzung von Studenten/-innen an der TU Darmstadt unter besonderer Berücksichtigung des mobilen Fremdsprachenlernens mit Podcasts empirisch untersuchen. In den folgenden Abschnitten widmen wir uns zunächst den wichtigsten Definitionen, Merkmalen und Formen mobilen Fremdsprachenlernens sowie dem aktuellen Forschungsstand zum Fremdsprachenlernen mit Podcasts. Anschliessend stellen wir die Konzeption unserer Studentenbefragung dar und präsentieren ausgewählte relevante Befragungsergebnisse in Bezug auf die Nutzung von Podcasts zum Fremdsprachenlernen. Daraus ziehen wir im letzten Teil Schlussfolgerungen für denkbare Einsatzmöglichkeiten von Podcasts innerhalb und ausserhalb des Fremdsprachenunterrichts.

\section{Definitionen, Merkmale und Formen mobilen Fremdsprachenlernens}

Der Terminus «mobiles Lernen» (auch «Mobile Learning» oder auch «M-Learning»), der eine sinnvolle Weiterentwicklung des Begriffs E-Learning darstellt, hat zurzeit noch keine eindeutige Definition. ${ }^{2}$

In unserer Arbeitsdefinition verwenden wir das mobile Lernen als umfassenden Sammelbegriff für alle Lehr- und Lernvorgänge, die mit Unterstützung jeder Zeit verfügbarer, transportabler und zumeist vernetzter Endgeräte (wie handflächen-

1 Dies hängt nicht nur mit der stetig voranschreitenden Computertechnologie, sondern auch mit der Tatsache zusammen, dass mobile Lernarrangements sehr komplexe und anspruchsvolle didaktische Handlungsfelder darstellen, die sich schwierig mit wissenschaftlichen Erkenntnissen sichern lassen.

2 Einen Überblick über verschiedene Definitionen liefern Traxler (2009), Bartelsen (2011, 2 ff.) und Rohs $(2013,78)$. Zu den Kernfragen der neueren Forschungsarbeiten vgl. Specht, Kalz und Börner (2013, 56). 
grosse Mobiltelefone und sich in ihrer Funktionalität permanent erweiternde multimediafähige Smartphones ${ }^{3}$ ) ablaufen und die E-Learning Komponente ausserhalb und innerhalb üblicher Lernorte nutzbar machen.

Das mobile Lernen lässt sich anhand folgender Merkmale charakterisieren:

1. Mobiles Lernen geht von einem lernerzentrierten, selbstbestimmten und output-orientierten konstruktiven Lernmodell aus.

2. In allen Konzepten mobilen Lernens nehmen die zentralen didaktisch-methodischen Prinzipien wie Authentizität, Kontextbezogenheit und Situiertheit ${ }^{4}$ von Lernanlässen grösstmöglichen Raum ein (Informationen sollen in einem authentischen Kontext dargeboten werden).

3. Vielfältige Formen mobilen Lernens fokussieren sowohl auf ein selbstgesteuertes und individuelles als auch ein kooperatives und kollaboratives Lernen, das «die bedarfsorientierte Bereitstellung von Informationen, die Verbindung zu den persönlichen sozialen Netzwerken und die Einbettung in formale Lernangebote» (Rohs 2013, 85) ermöglicht.

4. Mobiles Lernen bietet realitätsnahe sowie zeitlich und örtlich flexible Unterrichtsgestaltung und ermöglicht, unmittelbare und uneingeschränkt verfügbare Lerninhalte auch in Form interaktiver Lerneinheiten einzubinden (vgl. Mayrberger 2013, $5 \mathrm{ff}$.$) .$

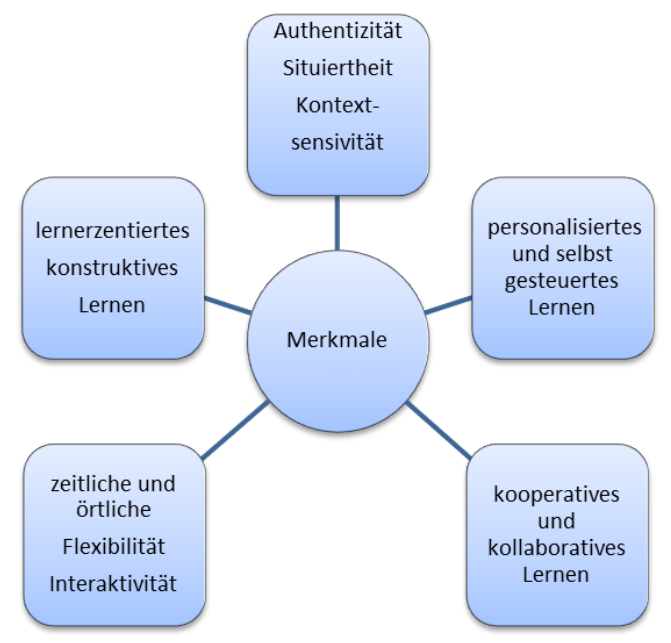

Abbildung 1: Merkmale mobilen Lernens

3 Mittlerweile (z. B. bei Herber und Waba 2011; de Witt 2013, 14) fallen darunter auch DIN A4 grosse Geräte wie Netbooks oder Tablet PCs, aber auch Navigationsgeräte, portable mp3- oder mp4Geräte. Mitschian $(2010,13)$ rechnet aber auch Computer mit normalen Ausmassen dazu. Zu den verschiedenen Funktionen von Endgeräten als Informationsquelle, Kommunikationsmedium und kognitives Werkzeug vgl. de Witt $(2013,18)$.

4 Rensing und Tittel $(2013,122)$ verstehen situiertes mobiles Lernen als «die Teilmenge von kontextualisiertem mobilen Lernen, in der ein enger Bezug zwischen der physischen Umgebung des Lernenden und seinen Lerninhalten besteht». 
5. Mobiles Lernen überwindet die Grenzen des formalen Unterrichts und dringt in die persönliche Lernumgebung des Lerners, der laut Herber und Waba (2011, 2) als Impulsempfänger, Weltentdecker, Forscher und Netzwerker auftritt ${ }^{5}(\mathrm{vgl}$. Abbildung 1).

Die in unserem Beitrag weit gefassten Kategorien mobilen Lernens mit Podcasts, die sowohl in formalen (präsenzgebundenes mobiles Lernen) als auch in informellen ${ }^{6}$ (selbstgesteuertes mobiles Lernen, ortsunabhängiges Lernen mit mobilen Endgeräten) sowie gemischten Lernszenarien Anwendung finden, erfolgen in verschiedenen Formen, die im Folgenden tabellarisch kurz charakterisiert werden.

\begin{tabular}{|c|c|c|}
\hline Ortsbasiert & Personalisiert & Kombiniert \\
\hline $\begin{array}{l}\text { Lernszenarien, die mit eher } \\
\text { formalisiertem Kontext } \\
\text { (d. h. unter Anwesenheit } \\
\text { einer Lehrperson in Grup- } \\
\text { pen- oder Einzelarbeiten) } \\
\text { realisiert werden, in denen } \\
\text { Lehrer/innen über portable } \\
\text { Abspielgeräte den Hörtext } \\
\text { in Form eines Podcasts mit } \\
\text { individuellem Hörtempo } \\
\text { bearbeiten, die Rezeption } \\
\text { bzw. die Produktion von } \\
\text { Podcasts mit den vom } \\
\text { Computer losgelösten } \\
\text { Unterrichtsschritten ver- } \\
\text { knüpfen und die Möglich- } \\
\text { keit der direkten sozialen } \\
\text { Interaktion mit authenti- } \\
\text { schen Sprechanlässen in die } \\
\text { Wege leiten. }\end{array}$ & $\begin{array}{l}\text { Einsatzszenarien, in denen } \\
\text { die Lerner/innen über por- } \\
\text { table Abspielgeräte von je- } \\
\text { dem beliebigen Ort Zugriff } \\
\text { auf Podcasts haben und ihr } \\
\text { Hörtraining individuell, in- } \\
\text { formell und selbst gesteuert } \\
\text { bestimmen. }\end{array}$ & $\begin{array}{l}\text { Gemischte (formale und } \\
\text { informelle) Lernsettings, } \\
\text { in denen die Lerner/innen } \\
\text { einen differenzierten (d. h. } \\
\text { stationären, mobilen, grup- } \\
\text { penorientierten, individu- } \\
\text { ellen) Zugriff auf Podcasts } \\
\text { haben und zu verschiede- } \\
\text { nen Lernaktivitäten über } \\
\text { die Kommentarfunktion der } \\
\text { vielen Podcast-Anbieter } \\
\text { (z. B. in Form eines Blogs) } \\
\text { angeregt werden. }\end{array}$ \\
\hline
\end{tabular}

Noch stehen allerdings die Beschreibungen konkreter Lernszenarien aus. Mitschian $(2010,120 \mathrm{f}$.) deutet zwei Möglichkeiten an, die «auf Rezeption eines vorgefertigten Lernangebots ausgerichtete Lernerhaltung» (Mitschian 2010, 120) zu durchbrechen, die allerdings noch Zukunftsmusik sind: Zum einen durch aktivierende Übungsformen, bei denen die Lerner/innen Eingaben über das Display machen oder mündlich reagieren. Zum anderen durch die Bildung virtueller Gruppen oder

5 Zur genauen Charakteristik der vier genannten Dimensionen des mobilen Lernens aus Sicht der Lerner/innen vgl. Herber und Waba $(2011,3)$.

6 Rohs $(2013,82)$ fasst mobiles informelles Lernen als «ein situatives, intrinsisch motiviertes, selbst bestimmtes und ortunabhängiges Lernen mit digitalen Medien» auf. 
Lernertandems, wobei sich diese kooperativ mit den Podcasts als fremdsprachlichen Inputs beschäftigen. Soweit die methodisch-didaktischen Überlegungen zum mobilen Lernen mit Podcasts. Im nächsten Abschnitt werden wir darauf eingehen, welche empirischen Forschungen es zum Fremdsprachenlernen mit Podcasts bisher gibt.

\section{Aktueller Forschungsstand zum Fremdsprachenlernen mit Podcasts}

Den Begriff «Podcast» gibt es seit mehr als zehn Jahren, weshalb es wohl bedeuten würde, Eulen nach Athen zu tragen, wenn wir - noch dazu in der Zeitschrift Medienpädagogik - das Phänomen «Podcast» langatmig vorstellen. Deshalb nur kurz einige Worte dazu (vgl. im Folgenden Stork 2012, 4). Unter Podcasts im weiteren Sinne verstehen wir Audio- oder Videoaufnahmen, die - in der Regel kostenlos - aus dem Internet herunterladbar sind. Im engeren Sinne verstehen wir darunter Audiooder Videoaufnahmen, die regelmässig erscheinen und abonniert werden können. Inzwischen gibt es in der Fachliteratur zur Fremdsprachenforschung eine Reihe von Veröffent-lichungen zu Erfahrungsberichten, zum technischen und didaktischen Potenzial sowie zu methodisch-didaktischen Vorschlägen zur Arbeit mit Podcasts im Fremdsprachenunterricht.? Und zwar sowohl in Bezug auf die Rezeption von Podcasts als auch auf die Produktion von Podcasts. Forschungsarbeiten in Bezug auf Podcasts beim Fremdsprachenlehren und -lernen jedoch sind (noch) rar gesät. Im Folgenden stellen wir die wenigen publizierten deutschsprachigen Studien kurz vor und zeigen im Anschluss daran den Ansatzpunkt unserer eigenen Studie auf. Rüdiger Schreiber (2010) hat eine Aktionsforschung zum Einsatz von Podcasts und MP3 im Deutsch als Fremdsprache-Unterricht an einem Studienkolleg durchgeführt. Ziel seines Projektes war es, studentische Präsentationen zu unterstützen, die Aussprache individuell zu fördern und das Hörver-stehen mit mündlichen Hausaufgaben zu verknüpfen (Schreiber 2010, 191). Die Lerner/innen sollten als Hausaufgabe Fragen zu einem Videopodcast mündlich mit MP3-Auf $\neg$ nahmen beantworten und an den Lehrer schicken. Dieser erstellte - ebenfalls als MP3 - eine individuelle Korrekturaufnahme für jeden Studenten. Der Student hatte die Möglichkeit, seinerseits eine zweite Aufnahme zu erstellen und wiederum dem Lehrer einzureichen. In den Evaluationsbögen bewerteten die Studenten/-innen das Projekt positiv, wobei das Gefühl beim freien Sprechen besser war als die Selbstwahrnehmung tatsächlicher Verbesserungen der Aussprache (Schreiber 2010, 207). Eine Analyse der vorliegenden Zweitfassungen der Aufnahmen der Studenten/innen zeigt, dass die Beiträge bei regelmässiger Mitarbeit nachhaltig besser wur-

7 Mit dem Potenzial von Podcasts für die Schulung des Hörverstehens in der Fremdsprache beschäftigt sich beispielsweise Franziska Emde (2012). Sie widmet ihren Beitrag englischsprachigen Podcasts und gibt konkrete Ideen für die Verwendung von Podcasts zur Schulung des Hörverstehens im Englischunterricht. Dabei spricht sie sich für die selbstständige Verbesserung der HV-Kompetenz mit Podcasts aus (vgl. Emde 2012, 71). 
den (Schreiber 2010, 205). Allerdings war insgesamt die Quote der Zweitfassungen von MP3-Aufnahmen nach erfolgter Lehrerkorrektur im Laufe des Projekts stark rückläufig.

Torben Schmidt und Kristina Peuschel beleuchten in ihren Forschungsprojekten den Aspekt der Veröffentlichung von Podcasts bzw. genauer gesagt die kulturelle Teilhabe, die dadurch ermöglicht wird. Es handelt sich dabei um die beiden grossen Forschungsarbeiten, die in Deutschland zu fremdsprachigen Podcasts durchgeführt wurden bzw. werden.

Schmidt führt seit 2007 ein Habilitationsprojekt durch zum Einfluss der Veröffentlichung von Podcasts im Internet auf die Motivation der Lerner/innen, ihr situationales Sachinteresse, die fremdsprachliche Qualität der erstellten Beiträge und auf die Identifikationen von Schülern/-innen der Sekundarstufe II mit der Fremdsprache als Kommunikationsmedium (vgl. Schmidt 2009, 32). In einem Aufsatz stellt Schmidt ausgewählte Ergebnisse seiner explorativ-interpretativ angelegten Unterrichtsbegleitforschung vor. Er zeigt, dass Öffentlichkeit und Publikum durch die Veröffentlichung von Podcasts Anreiz und Verpflichtung für die Lerner/innen darstellten und somit einen positiven Einfluss auf ihre Mo $\neg$ tivation hatten (vgl. Schmidt 2009, 36 ff.). Andererseits räumt er aber auch ein, dass der für die Projektarbeit benötigte Zeitaufwand eine grosse Belastung darstellte und viele Schüler/ innen die Unvereinbarkeit mit dem Regelunterricht kritisierten (Schmidt 2009, 39). Kristina Peuschel (2012a) hat ihre Dissertationsschrift vorgelegt, in der sie in einer longitudinalen, explorativ-interpretativen Studie die sprachlichen Handlungsprozesse von Deutschlernern/-innen beschreibt, die Radiosendungen und Podcasts erstellt haben. Für das Lernen in sog. «radiodaf-Projekten» hat sie abschliessend vier Teilhabeoptionen rekonstruiert, und zwar:

- "die Teilhabe der Lernenden an ihrer Projektgruppe im je spezifischen Sprachlernkontext (T1),

- die Teilhabe an der face-to-face Kommunikation mit Sprechenden der Zielsprache im Projektverlauf (T2),

- die imaginierte Teilhabe an der massenmedial vermittelten Kommunikation mit der Zielsprachengesellschaft während des Erstellens der Vorlagen und des mündlichen Realisierens (T3),

- die abschliessende Teilhabe an der massenmedial vermittelten Kommunikation mit der Zielsprachengesellschaft (T4).» (Peuschel 2012a, 202). Zu weiteren Ergebnissen vgl. Peuschel (2007, 2009a, 2009b, 2012b).

Beforscht wurden somit bisher beispielhafte Projekte ${ }^{8}$, in denen im Fremdsprachenunterricht Podcasts für eine kleinere oder grössere Öffentlichkeit erstellt

8 Morten Hunke (2011) berichtet beispielsweise über ein interkulturelles Projekt, in dem englische Studenten/-innen im Auslandsjahr im deutschsprachigen Raum Podcasts zur Reflexion über ihre 
wurden. In den Blick genommen wurde hauptsächlich die Produktion von Podcasts und nicht so sehr die Rezeption von Podcasts. Wir haben in Deutschland zwar statistische Daten zur Nutzung von Podcasts (vgl. z. B. die Analysen in der Online-Fachzeitschrift Media Perspektiven) oder mit der Jim-Studie eine regelmässig durchgeführte Basisstudie zum Umgang von 12- bis 19-Jährigen mit Medien und Information. Es liegen aber bisher im deutschsprachigen Raum keine Daten zur Nutzung von fremdsprachigen Podcasts vor. In Polen sieht die Forschungslage dazu dank der deutschsprachigen Dissertationsschrift von Katarzyna Trojan (2014), die sich mit dem Einsatz von Podcasts bei der Förderung fremdsprachlichen Hörverstehens am Beispiel polnischer Studenten/-innen der Germanistik und angewandter Linguistik beschäftigt, besser aus. Die von der Autorin durchgeführte Studie hat u.a. ergeben, dass Podcasts nach der Ansicht von 47,1\% der befragten polnischen Studenten/-innen im deutschsprachigen Unterricht eingesetzt werden. Bei der selbstständigen Förderung der HV-Kompetenz ausserhalb des Unterrichts werden Podcasts zwar von über der Hälfte der Befragten (53,7\%) gehört, wobei nur von 9,8\% der Studenten/-innen regelmässig. Studenten/-innen hören Podcasts deutlich häufiger traditionell zu Hause am Computer als unterwegs auf einem mobilen Gerät wie z. B. Smartphone oder MP3-/MP4-Player. Mitschian (2010, 135) stellt angesichts der Möglichkeiten des mobilen Lernens kritisch die Frage, «wer überhaupt immer und überall lernen will» und ob Handys oder MP3-Player als mobile Lernmedien Akzeptanz finden.

Angesichts des Forschungsdesiderats in Bezug auf die Rezeption von fremdsprachigen Podcasts im deutschsprachigen Raum halten wir es für notwendig, zu erforschen, ob und wie Podcasts von Studenten/-innen zum Fremdsprachenlernen genutzt werden. Denn nur auf der Grundlage dieser empirischen Ergebnisse können darauf abgestimmte didaktisch-methodische Empfehlungen entwickelt werden.

\section{Fragestellungen, Konzeption und Beteiligte der Studentenbefragung an der TU Darmstadt}

Im Folgenden erläutern wir Fragestellungen, Konzeption und Auswertung unserer explorativ angelegten Fragebogenstudie zum mobilen Fremdsprachenlernen mit Podcasts, stellen des Weiteren ausgewählte relevante Ergebnisse dar und analysieren sowie diskutieren diese.

Die Untersuchung im Rahmen des Forschungsprojektes zum mobilen Fremdsprachenlernen setzte sich u. a. zum Ziel, folgenden Forschungsfragen nachzugehen:

- (Wie) Werden Podcasts allgemein genutzt?

Lernprozesse genutzt haben. Sie waren verpflichtet, im Rahmen des Projekts einerseits selbst Reflexionspodcasts zu produzieren, in denen sie die während des Aufenthalts erworbenen Fähigkeiten reflektiert haben, andererseits Podcasts ihrer Kollegen anzuhören und zu kommentieren (vgl. Hunke 2011, $299 \mathrm{ff}$.$) .$ 
- (Wie) Werden Podcasts zum Fremdsprachenlernen genutzt?

- Welche Wünsche gibt es zur Nutzung von Podcasts zum Fremdsprachenlernen?

Den Forschungsfragen lag die Annahme zugrunde, dass mobile (personalisierte und kombinierte) Formen des Fremdsprachenlernens von den Studenten/-innen nur begrenzt genutzt werden. Darüber hinaus wurde angenommen, dass Podcasts nicht allgemein bekannt sind und dadurch beim Fremdsprachenlernen nur selten eingesetzt werden. Die Mehrheit der Studenten/-innen weiss nicht, wie sie ihre Fremdsprachenlernprozesse mit Podcasts unterstützen könnte.

Die Daten zu den gestellten Fragen wurden mittels eines schriftlichen Fragebogens erhoben. Die Befragung in Form eines Fragebogens bot uns die Möglichkeit, eine grössere Probandenzahl im Bereich mobilen Fremdsprachenlernens mit Podcasts aus externer Perspektive zu untersuchen und dabei zu generalisierbaren Ergebnissen zu kommen. Die Erhebung wurde Anfang Februar 2013 am Sprachenzentrum der Technischen Universität (TU) Darmstadt unter Studenten/-innen in Deutsch-als-Fremdsprache-Kursen sowie Studenten/-innen in Englischkursen durchgeführt. Insgesamt konnten nach dem Rücklauf 144 Fragebögen statistisch ausgewertet werden.

Der Fragebogen setzte sich aus vier Fragenkomplexen zusammen und umfasste 21 Fragen (zum Aufbau des Fragebogens vgl. den Anhang). Bei den 144 Befragten handelte es sich um Studenten/-innen in Deutsch-als-Fremdsprache-Kursen ${ }^{9}$ (GER-Niveaus B1, B2 und C1) sowie Studenten/-innen in unterschiedlichen Englischkursen ${ }^{10}$ (GER-Niveaus B1, B2 und C1) am Sprachenzentrum der TU Darmstadt. Befragt wurden 47 Frauen (33\%) und 97 Männer (67\%) im Alter von 18 bis 34 Jahren unterschiedlicher Herkunft, von Albanern bis hin zu Vietnamesen, wobei die Mehrheit diejenigen bildeten, deren Erstsprache Deutsch war (32\%). An zweiter Stelle platzierte sich als Erstsprache der Befragten Chinesisch (12\%), gefolgt von Spanisch (4\%) an dritter Stelle.

\section{Bestandsaufnahme zum Einsatz von Podcasts zum Fremdsprachenlernen Art und Umfang der Podcastnutzung allgemein}

Mit Hilfe von drei Fragestellungen sollte ermittelt werden, ob, wie oft und wozu Podcasts von den Befragten genutzt werden. Aus Platzgründen wird nur auf die Antworten auf eine Frage eingegangen, und zwar auf die Frage nach der Häufigkeit der Nutzung von Podcasts.

9 Dabei handelte es sich um folgende Deutschkurse aus dem Angebot des Sprachenzentrums an der TU Darmstadt: Deutsche Sprachprüfung für den Hochschulzugang, Grammatik II, Lesen-VerstehenDiskutieren II, Deutschkurs

10 Die Befragten beteiligten sich an folgenden Englischkursen aus dem Angebot des Sprachenzentrums der TU Darmstadt: English-speaking Booster, Advanced general language course, General English course for science and technology, General language course for science and technology, Englischkurs, General English course. 
Aus den Antworten geht deutlich hervor, dass Podcasts von fast einem Viertel der Probanden (24\%) nie genutzt werden. Diejenigen, die Podcasts hören (75\%), tun das vorwiegend manchmal (54\%). Oft werden Podcasts nur von 15\% der Probanden genutzt. Nur 6\% der Studenten/-innen nutzen Audio- bzw. Videodateien, die im Internet zur Verfügung stehen sehr oft. Diese Ergebnisse waren recht erfreulich. Sie bezogen sich jedoch nur auf die allgemeine Nutzung von Podcasts. Uns interessierte im Anschluss daran die Frage, ob Podcasts von den Befragten auch zum Fremdsprachenlernen genutzt werden.

\section{Art und Umfang der Podcastnutzung zum Fremdsprachenlernen}

Um zu erfragen, ob, wie und welche Podcasts beim Fremdsprachenlernen genutzt werden, waren folgende Fragestellungen im dritten Fragenkomplex von besonderem Interesse:

- Hören Sie Podcasts zum Fremdsprachenlernen?

- Hören Sie Podcasts mobil?

- Welche Podcasts zum Fremdsprachenlernen hören Sie?

- Würden Sie gerne ausserhalb des Klassenraumes oder im Klassenraum Podcasts

hören?

- Welche Art von Podcasts zum Fremdsprachenlernen bevorzugen Sie?

Aus den Antworten auf die erste Frage dieses Fragenkomplexes geht hervor, dass fast die Hälfte der Befragten (49\%) keine Podcasts zum Fremdsprachenlernen nutzt. Manchmal werden Podcasts von 35\% der Befragten gehört. Oft hören nur etwa $10 \%$ der Befragten Podcasts. Dabei werden Podcasts nur von $24 \%$ der

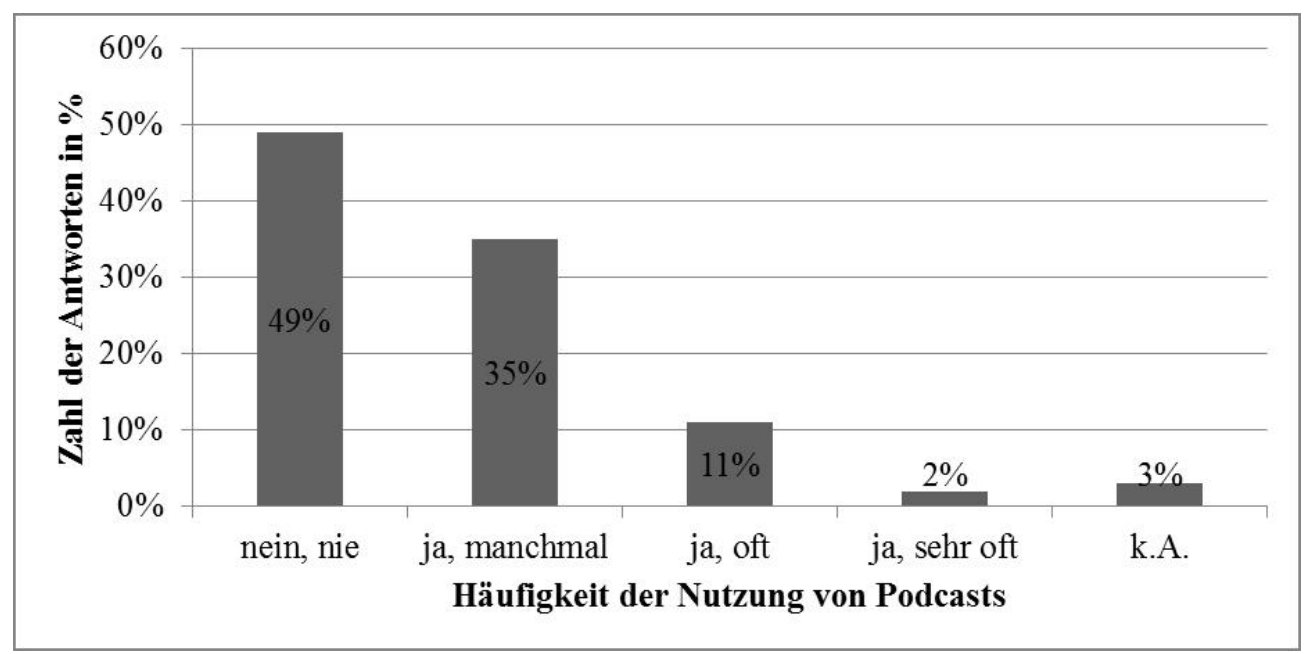

Abbildung 2: Häufigkeit der Nutzung von Podcasts zum Fremdsprachenlernen 
Befragten mobil gehört. Diese Angaben korrespondieren grösstenteils mit den Antworten auf die Frage nach der Nutzung mobiler Geräte beim Fremdsprachenlernen allgemein. Hier zeigt sich, dass Podcasts zwar mit mobilem Lernen in der Theorie gleichgesetzt werden, ihre Nutzung mithilfe von portablen Abspielgeräten in der Praxis jedoch nicht selbstverständlich ist.

Mit der nächsten Frage sollte eruiert werden, welche fremdsprachigen Podcasts gehört werden. Bei den von den Befragten gehörten deutschsprachigen Podcasts sind Podcasts der Deutschen Welle klare Favoriten (16 Nennungen), darunter z. B. Langsam gesprochene Nachrichten und Jojo sucht das Glück sowie Deutsch im Alltag. Des Weiteren hören die Befragten gerne ZDF-Nachrichten (5 Nennungen) und Slow German (4 Nennungen). Gelegentlich werden Podcasts auf den Seiten anderer deutscher Radio- und Fernsehsender gehört, z. B. solche Podcasts wie diejenigen von Dradio, Podcasts des Hessischen Rundfunks, Tageschau in 100 Sekunden, Sesamstrasse. Was die englischsprachigen Podcasts anbelangt, bevorzugen Studenten/-innen vor allem BBC- (6 Minutes English), CNN-, und Leo-Podcasts. 16 Befragte haben keine Titel angegeben. Die fehlenden Angaben zu den Titeln von gehörten Podcasts könnten ein Indiz für einen mangelnden Bekanntheitsgrad von Podcasts bei den Befragten sein.

Bei der Beantwortung der nächsten Frage listen die Befragten ein paar Bedingungen auf, die erfüllt werden sollten, damit sie Podcasts zum Fremdsprachenlernen hören. Sie lassen sich in folgenden Stichpunkten zusammenfassen (In Klammern werden zur Verdeutlichung einige Zitate herangezogen):

- mehr Zeit («Wenn ich genug Freizeit hätte» (FB. 12), «Nicht zu zeitintensiv» (FB. 141)) (7 Nennungen),

- freier Zugang/ kostenlos («Frei zugänglich/ keine Kosten» (FB. 66), «einfacher Zugang, niedrige/keine Kosten» (FB. 139)) (5 Nennungen),

- Interesse («wenn interessant» (FB. 135), «interessante Themen» (FB. 140), «spassige Apps» (FB. 136)) (5 Nennungen),

- Empfehlung ( $« W e n n$ mir jemand bestimmte Podcasts zum Fremdsprachenlernen empfehlen würde» (FB. 90), «Wenn sie Teil des Unterrichts wären oder als Tipp des Lehrers kommen würden» (FB. 126)) (4 Nennungen),

- Anpassung an das sprachliche Niveau («lch habe nichts genug Niveau» (FB. 24), «bessere Angebote auf meinem Level» (FB. 147)) (3 Nennungen),

- Authentizität («müssen von einem Native-Speaker gesprochen sein» (FB. 91)) (1 Nennung)

- Motivation («Wenn ich mehr motiviert wäre» (FB. 117)) (1 Nennung),

- Hilfe bei der Auswahl («Es ist schwer gute Podcasts zu finden» (FB. 99)) (1 Nennung).

Die folgende Abbildung soll zeigen, wo Studenten/-innen Podcasts gern hören würden. 


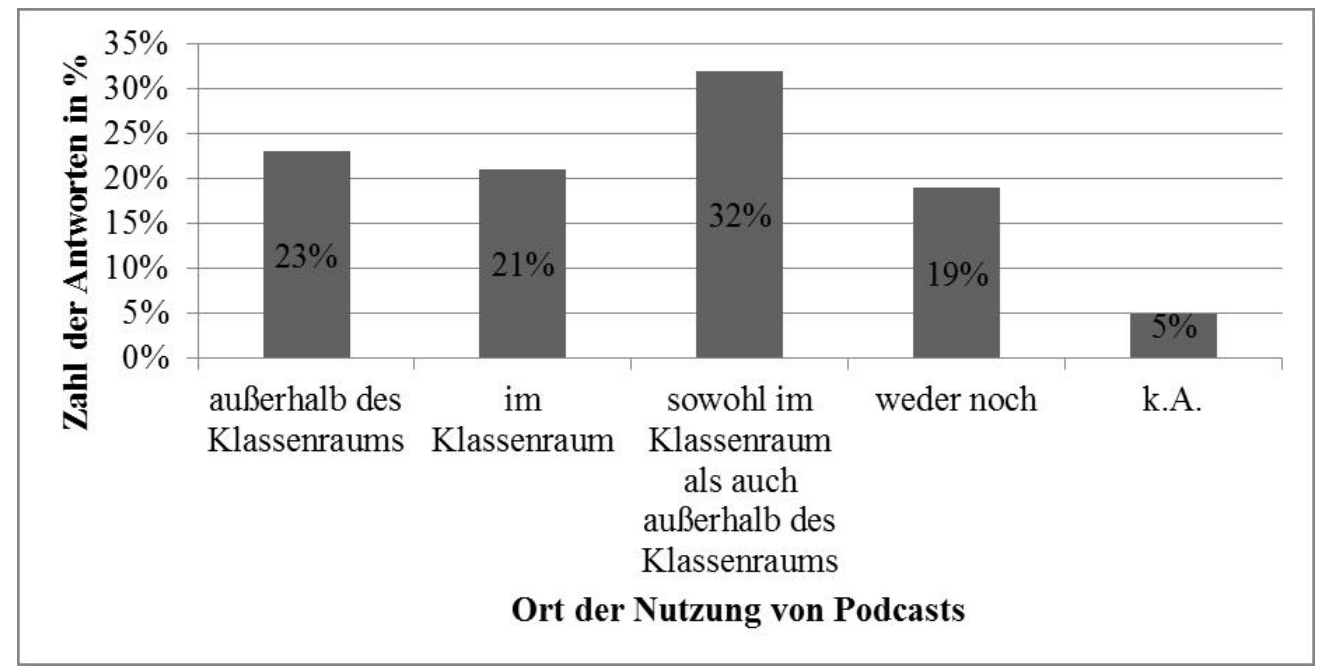

Abbildung 3: Ort der Nutzung von Podcasts

Die Abbildung ergibt, dass fast ein Drittel der Beteiligten (32\%) Podcasts sowohl im Klassenraum als auch ausserhalb des Klassenraums hören würde. Die Aussagen der Studenten/-innen verdeutlichen, dass sie eine kombinierte Form mobilen Lernens (vgl. Abbildung 2) bevorzugen würden. Besonders auffällig ist jedoch die Zahl der Antworten in der Kategorie "weder noch», was die Schlussfolgerung erlaubt: Fast 20\% der Studenten/-innen wollen überhaupt keine Podcasts hören. Der Grund dafür könnte in der mangelnden Kenntnis der Vorteile mobilen Lernens, vielmehr aber in der unzureichenden Erfahrung mit der Arbeit mit Podcasts beim Fremdsprachenlernen liegen.

Die Befunde der Frage zur bevorzugten Art von Podcasts sind der folgenden Abbildung zu entnehmen.

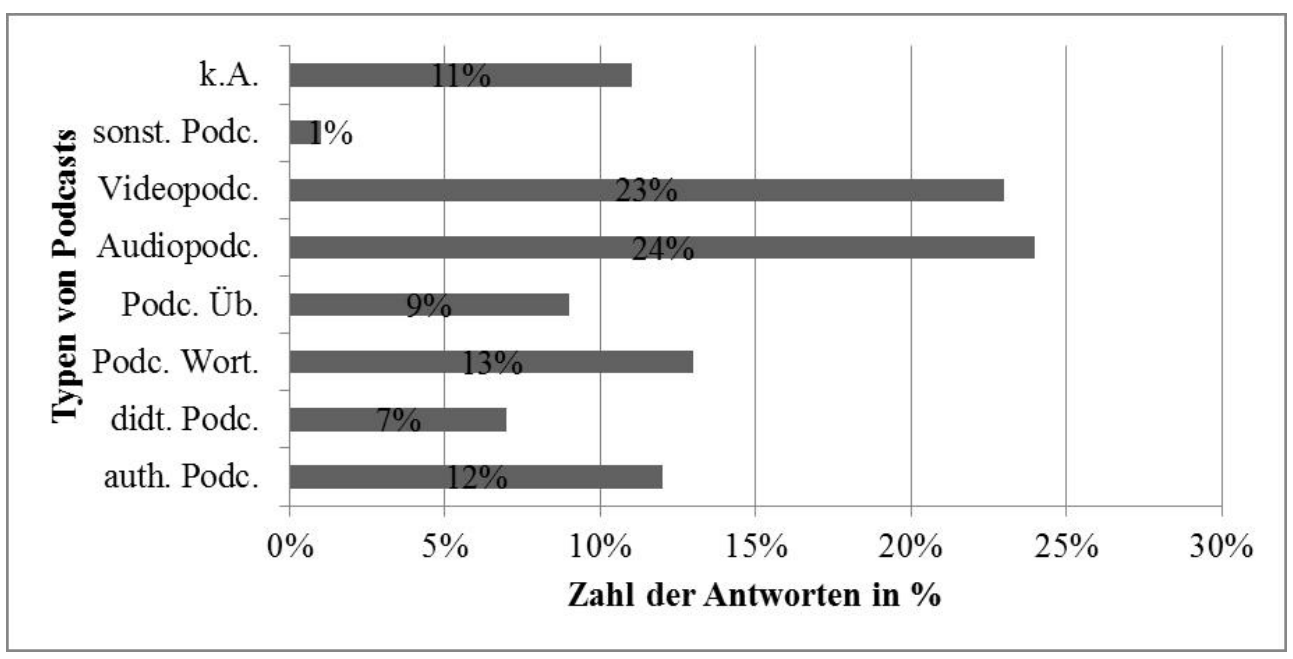

Abbildung 4: Typen von Podcasts 
Betrachtet man die Ergebnisse der obigen Darstellung, so lässt sich schlussfolgern, dass Studenten/-innen sowohl Audiopodcasts als auch Videopodcasts hören bzw. sehen wollen. Was den Didaktisierungsgrad von Podcasts angeht, dann wählen 13\% der Befragten Podcasts mit schriftlichen Wortschatzerklärungen. Nur 9\% der Befragten bevorzugen Podcasts mit Übungen zum Podcasttext. Vermutlich ist dies so, weil sie nicht wissen, dass es solche didaktisierten Podcasts gibt oder sie hören Podcasts auf dem Weg zur Universität, zum Sprachenkurs und haben keine Zeit und/oder keine Möglichkeit zusätzlich noch Übungen zum Text zu lösen.

\section{Adressatengerechte Wünsche zur Nutzung von Podcasts zum Fremdsprachenlernen}

Zum Schluss sollte der Frage nachgegangen werden, welche Hilfestellungen Befragte brauchen, um Podcasts zum Fremdsprachenlernen zu hören. Die Aussagen der Teilnehmer/innen ergeben die folgende Übersicht:

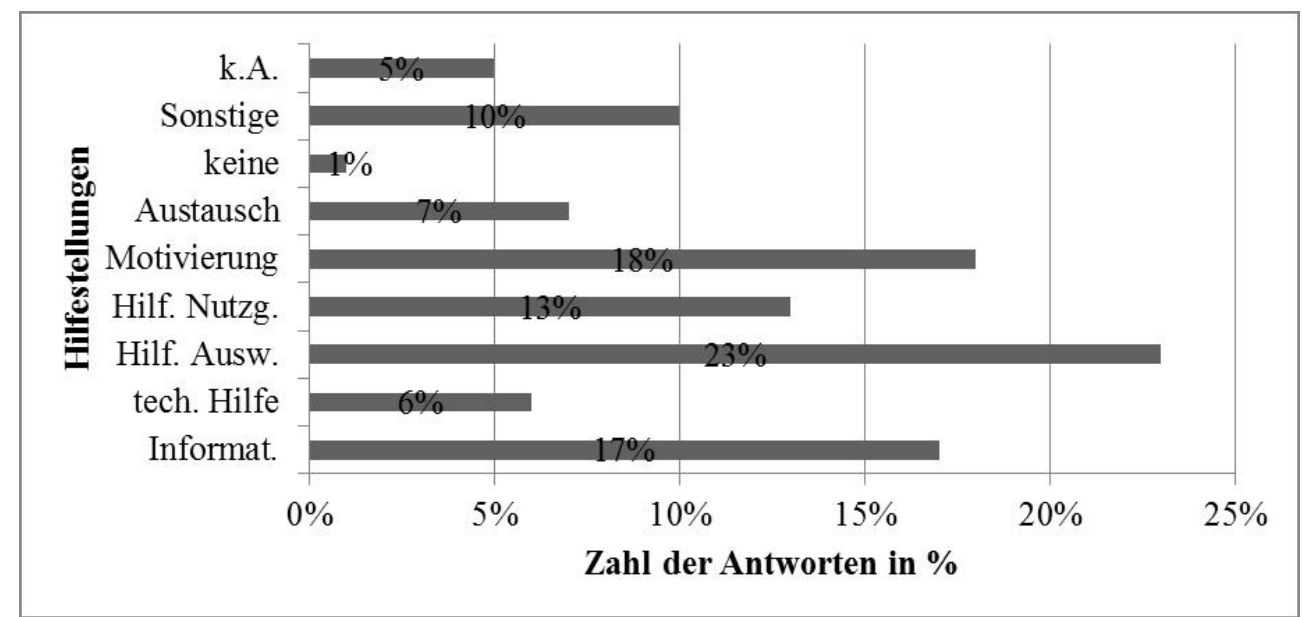

Abbildung 5: Wünsche zur Nutzung von Podcasts

Besonders auffällig ist im Kontext der vorliegenden Untersuchung der Sachverhalt, dass nur $1 \%$ der Befragten keine Hilfe beim Umgang mit Podcasts beim Fremdsprachenlernen braucht. Studenten/-innen brauchen Hilfe vor allem bei der Auswahl von Podcasts (23\%). Darüber hinaus werden Motivierung von der Kursleitung (18\%) sowie weitere Informationen (didaktisch-methodische Hinweise zum Fremdsprachenlernen mit Podcasts) (17\%) als notwendige Hilfestellungen genannt.

Die Befunde zeigen mit aller Deutlichkeit, dass fast alle Befragten Hilfe bei der Nutzung von Podcasts beim (mobilen) Fremdsprachenlernen brauchen. Vor dem Hintergrund der bereits diskutierten Befunde sind diese Erkenntnisse jedoch nicht verwunderlich. Sie bestätigen unsere Annahme, dass nur wenige Studenten/-innen wissen, wie sie mit Podcasts ihr Fremdsprachenlernen unterstützen könnten. 


\section{Schlussfolgerungen und Ausblick mit Konsequenzen für mögliche Anwen- dungsszenarien innerhalb und ausserhalb des Fremdsprachenunterrichts}

Die Antworten der Befragten zur Nutzung von Podcasts beim Fremdsprachenlernen bekräftigen unsere Annahme, dass Podcasts eher selten beim Fremdsprachenlernen bei den befragten Studenten/-innen Anwendung finden (49\%), was im Zeitalter des mobilen Lernens verwundern mag. Die Erklärung der oben angeführten Befunde liegt nach Meinung der Autorinnen darin, dass Studenten/-innen keine Zeit haben, um Podcasts zu hören, sich dessen nicht bewusst sind, dass das Internet eine riesige Materialfülle an fremdsprachigen Podcasts bietet, die meist kostenlos verfügbar sind. Sie wissen auch nicht, dass sie Podcasts abonnieren können und orts- und zeitunabhängig hören können.

Die Ergebnisse zu eventuellen Hilfestellungen bei der Nutzung von Podcasts geben Aufschluss darüber, dass fast alle Studenten/-innen Hilfe bei der Nutzung von Podcasts beim (mobilen) Fremdsprachenlernen brauchen. Mit Blick darauf erscheint es ratsam, eine in dieser Hinsicht durchdachte didaktisch-methodische Konzeption des mobilen Fremdsprachenlernens mit Podcasts zu entwickeln.

Aus den oben zusammengefassten und diskutierten Forschungsergebnissen, deren Reichweite und Verallgemeinerbarkeit in einer grösseren kontrollierten Untersuchung unter Fremdsprachenlernenden in unterschiedlichen Altersgruppen noch überprüft werden sollte, ergeben sich wichtige didaktische Konsequenzen für mobiles Fremdsprachenlernen von Studenten/-innen mit Podcasts.

Etwa die Hälfte der Teilnehmer/innen wünscht sich, innerhalb des Fremdsprachenunterrichts bzw. innerhalb des Klassenraums Podcasts zu hören. Hier sind entsprechende didaktisch-methodische Konzepte zu entwickeln, und zwar sowohl was den Einsatz im Fremdsprachenunterricht betrifft als auch in Bezug auf eine Anleitung und Unterstützung der Lernenden beim eigenständigen Fremdsprachenlernen ausserhalb des Unterrichts. Dabei sind Audio- und Videopodcasts gleichermassen interessant. Die unterschiedliche Akzeptanz sowie die unterschiedlichen Präferenzen könnten für eine Individualisierung von Fremdsprachenlernprozessen sprechen. Hörtexte müssen mit mobilen Geräten nicht wie früher üblich als gemeinsame Aktivität mit der gesamten Klasse gehöert werden, sondern es ist eine Arbeit in Gruppen-, Partner- oder Einzelarbeit möglich. Die Lerner/innen können mit unterschiedlichen Podcasts (oder mit anderen Lernmedien) in ihrem eigenen Tempo unterschiedliche Aufgaben bearbeiten.

Ebenfalls etwa die Hälfte der Teilnehmer/innen präferiert das Hören von Podcasts ausserhalb des Fremdsprachenunterrichts bzw. ausserhalb des Klassenraums. Wer ausserhalb des Fremdsprachenunterrichts lernt und personalisierte oder kombinierte Formen mobilen Fremdsprachenlernens wählt, muss selbstständiges Lernen beherrschen. Er oder sie muss also wissen, wie Podcasts zum Fremdsprachenlernen genutzt werden können. Die Fragebogenergebnisse zeigen klar, dass die 
Studenten/-innen Hilfestellungen benötigen, und zwar bei der Auswahl von Podcasts, bei didaktisch-methodischen Möglichkeiten zum Fremdsprachenlernen mit Podcasts und sie brauchen eine Motivierung von der Kursleitung.

Dies legt nahe, dass im Fremdsprachenunterricht die Studenten/-innen zum Fremdsprachenlernen mit Podcasts befähigt werden sollten. Das heisst: Es sollte ihnen zunächst explizit erläutert werden, was Podcasts sind, wodurch sie sich auszeichnen, wo man sie beziehen kann, welche Möglichkeiten des Einsatzes sie anbieten und welche Fremdsprachenlernprozesse sie begünstigen können. Des Weiteren sollte den Studenten/-innen aufgezeigt werden, wie man selbstständig mit Podcasts an der Entwicklung von sprachlichen Fertigkeiten arbeiten kann. Auch Motivierungshilfen zur kontinuierlichen Arbeit mit Podcasts sollten besprochen werden. Die Anleitung zum mobilen Fremdsprachenlernen ist dabei ein Muss. Erst dann könnten sich die Studenten/-innen der Merkmale mobilen Lernens, wie z. B. der Authentizität, Lernerzentriertheit, Flexibilität, Interaktivität, der zeitlichen und örtlichen Unabhängigkeit, um nur einige Merkmale aufzulisten, bewusst werden. Unbestritten ist, dass Podcasts mobiles Fremdsprachenlernen stärken können. «Die Potenziale ergeben sich vor allem aus der grossen Vielfalt von Podcasts, ihrer Verfügbarkeit, Aktualität, Authentizität und unbegrenzter Thematik» (Trojan 2012,121).

\section{Literaturverzeichnis}

Bartelsen, Jan. 2011. «Mobile learning - ein Überblick.» Arbeitspapier der Nordakademie (2011-06): https://www.nordakademie.de/fileadmin/downloads/Arbeitspapiere/AP_2011_06.pdf.

Emde, Franziska. 2012. «Podcasts und Audiodateien zur Schulung des Hörverstehens: Beispiele für die Verwendung von Hörtexten im Englischunterricht.»In Web 2.0 im Fremdsprachenunterricht: Ein Praxisbuch für Lehrende in Schule und Hochschule, hrsg. v. Jürgen Wagner u. Verena Heckmann, 65-72. Glückstadt: Werner Hülsbusch.

Herber, Erich und Stephan Waba. 2011. «Lernen und Forschen mit mobilen Endgeräten - Wie finden wir Zugang zur persönlichen Umgebung der Lernenden?» Zeitschrift für e-Learning. Themenheft 4: 32-44.

Hunke, Morten. 2011. «Hamburg-Harburg ruft Leeds - Hallo, hier Leipzig ...) - Reflektierendes Podcasten von Studierenden während des obligatorischen Auslandsaufenthalts.» In Grenzen überwinden mit Deutsch: 37. Jahrestagung des Fachverbands Deutsch als Fremdsprache an der Pädagogischen Hochschule Freiburg/Br. 2010. (Materialien Deutsch als Fremdsprache; 85), hrsg. v. Natalia Hahn u. Thorsten Roelcke, 299-309. Göttingen: Universitätsverlag.

Mayrberger, Kerstin. 2013. «Unterwegs lernen. Mobile Endgeräte im Unterricht.» Praxis Fremdsprachenunterricht. Basisheft. Mobiles Lernen 1: 5-7. 
Mitschian, Haymo. 2010. m-Learning - die neue Welle? Mobiles Lernen für Deutsch als Fremdsprache. Kassel: kassel university press.

MMB-Institut für Medien und Kompetenzforschung. (2013). MMB Learning Delphi 2013: http://www.mmb-institut.de/monitore/trendmonitor/MMBTrendmonitor_2013_I.pdf.

Peuschel, Kristina. 2007. «Kommunikation und Teilhabe - Wie Deutschlernende in Radio- und podcast-Projekten zu medial präsenten Sprachnutzenden werden.» Deutsch als Zweitsprache 2: 33-41.

Peuschel, Kristina. 2009a. "Sprachliche Aktivitäten in Projekten - Radioprojekte für Deutsch als Fremdsprache aus der Lernendenperspektive.» In Qualitative Forschung in Deutsch als Fremdsprache, hrsg. v. Beate Baumann, Sabine Hoffmann u. Martina Nied, 107-121. Frankfurt am Main: Lang.

Peuschel, Kristina. 2009b. «Integrierte Textentwicklung in einem Radioprojekt mit fortgeschrittenen DaF-Lernenden.» In Kaleidoskop der jungen DaF-/DaZ-Forschung, hrsg. v. Kristina Peuschel u. Jan Paul Pietzuch, 89-106. Göttingen: Universitätsverlag.

Peuschel, Kristina. 2012a. Sprachliche Tätigkeit und Fremdsprachenlernprojekte: Fremdsprachliches Handeln und gesellschaftliche Teilhabe in radiodaf-Projekten. Baltmannsweiler: Schneider Verlag Hohengehren.

Peuschel, Kristina. 2012b. «Lerner-Podcasts - Präsentationen aus dem projektorientierten Unterricht.» Fremdsprache Deutsch 47: 30-34.

Praxis Fremdsprachenunterricht. 2013. Basisheft 01/13. Mobiles Lernen. Oldenbourg: Cornelsen.

Rensing, Christoph und Stephan Tittel. 2013. "Situiertes mobiles Lernen - Potenziale, Herausforderungen und Beispiele.» In Mobile Learning. Potenziale, Einsatzszenarien und Perspektiven des Lernens mit mobilen Endgeräten, hrsg. v. Claudia de Witt u. Almut Sieber, 121-142. Wiesbaden: Springer VS.

Rohs, Matthias. 2013. «Informelles Mobiles Lernen.» In Mobile Learning. Potenziale, Einsatzszenarien und Perspektiven des Lernens mit mobilen Endgeräten, hrsg. v. Claudia de Witt u. Almut Sieber, 75-97. Wiesbaden: Springer VS.

Schmidt, Torben. 2009. «Mündliche Lernertexte auf der 2.0-Bühne - Mediale Inszenierun $\neg$ gen im Englischunterricht am Beispiel eines Schulpodcast-Projekts.» Forum Sprache 1: 24-42.

Schreiber, Rüdiger. 2010. "Aktionsforschung zum Einsatz von Podcasts und mp3Dateien als Interaktionsmedium zwischen Dozenten und Lernenden.» In DaF integriert. Literatur - Medien - Ausbildung. 36. Jahrestagung des Fachverbands Deutsch als Fremdsprache an der Heinrich-Heine-Universität Düsseldorf 2008, hrsg. v. Matthias Jung u. Christoph Chlosta, 191-212. Göttingen: Universitätsverlag Göttingen. 
Specht, Marcus und Martin Ebner. 2011. «Mobiles und ubiquitäres Lernen: Technologien und didaktische Aspekte.» In Lehrbuch für Lernen und Lehren mit Technologien, hrsg. v. Sandra Schön u. Martin Ebner. http:///3t.tugraz.at/index. php/LehrbuchEbner10/article/view/74.

Specht, Marcus, Marco Kalz und Dirk Börner. (2013). «Innovation und Trends für Mobiles Lernen.» In Mobile Learning. Potenziale, Einsatzszenarien und Perspektiven des Lernens mit mobilen Endgeräten, hrsg. v. Claudia de Witt u. Almut Sieber, 55-74. Wiesbaden: Springer VS.

Stork, Antje. 2012. «Podcasts im Fremdsprachenunterricht - ein Überblick.» Informationen Deutsch als Fremdsprache 39 (1): 3-16.

Traxler, John. 2009. «Learning in a Mobile Age.» International Journal of Mobile and Blended Learning 1 (1): 1-12.

Trojan, Katarzyna. 2012. «Vom Podcastrezipienten zum Podcastproduzenten? Wie DaF-Studenten mit Hilfe von Podcasts Deutsch lernen können.» In Od jezyka do kultury, literatury, sztuki i mediów, hrsg. v. Marek Krawiec, 113-122. Wałbrzych: DTP Service.

Trojan, Katarzyna. 2014. Einsatz von Podcasts bei der Förderung fremdsprachlichen Hörverstehens am Beispiel polnischer Studierender der Germanistik und der Angewandten Linguistik. Unveröffentlichte Dissertation an der Adam-Mickiewicz-Universität Poznan.

de Witt, Claudia. 2013. «Vom E-Learning zum Mobile Learning - wie Smartphones und Tablet PCs Lernen und Arbeit verbinden.» In Mobile Learning. Potenziale, Einsatzszenarien und Perspektiven des Lernens mit mobilen Endgeräten, hrsg. v. Claudia de Witt u. Almut Sieber, 13-26. Wiesbaden: Springer VS. 